\title{
Splicing the MDS genome
}

Myelodysplastic syndrome (MDS) describes a range of haematological diseases that are associated with defective myeloid differentiation and dysplasia; in some cases, MDS progresses to acute myeloid leukaemia (AML). Three papers now present different aspects of a similar story: altered splicing can lead to MDS and even progression to AML.

To more clearly understand the pathogenesis of MDS in response to chronic DNA damage, Colla, Ong et al. used telomerase-deficient mice expressing inducible telomerase reverse transcriptase (Tert ${ }^{\mathrm{ER} / \mathrm{ER}}$ mice). These mice were then crossed with each other for multiple generations $(G)$ to degenerate their telomeres. G4 and G5 mice exhibited telomere dysfunction, DNA damage and tissue degeneration, and this phenotype was reversed by Tert induction. At 3 months, G4 and G5 mice exhibited refractory anaemia with excess blasts (RAEB; $80 \%$ of the cohort) or chronic myelomonocytic leukaemia (CMML; $20 \%$ of the cohort), both of which are types of MDS; $5 \%$ of these mice developed AML by 7 months.

Further analyses of G4 and G5 mice revealed that numbers of granulocyte-macrophage progenitors (GMPs) were significantly increased, megakaryocyte-erythroid progenitors (MEPs) were considerably reduced and common myeloid progenitors (CMPs; which produce GMPs and MEPs) were slightly reduced compared with age-matched controls. Additionally, ionizing radiation and cisplatin (an alkylating agent) induced impaired differentiation of wild-type CMPs into MEPs. Surprisingly, they found that inhibition of ataxia telangiectasia and Rad3-related (ATR), a DNA damage response kinase, in CMPs partially restored erythroid differentiation in clonogenic assays. Together, this indicates that telomere dysfunction induces an ATRmediated DNA damage response that elicits a differentiation checkpoint and altered CMP differentiation.

How does DNA damage skew CMP differentiation? The expression of RNA splicing genes, including Srsf2, was significantly reduced in G4 and G5 CMPs compared with CMPs from G0 Tert ${ }^{\mathrm{ER} / \mathrm{ER}}$ mice; this repression was reversed by Tert induction or by ATR inhibition. Importantly, haematopoietic cell-specific heterozygous knockout of Srsf 2 resulted in bone marrow dysplasia, myelomonocytosis, an increased frequency of GMPs, a decreased frequency of MEPs and altered RNA splicing in CMPs. Furthermore, CMPs from G4 and G5 mice had significantly more aberrant splicing events than CMPs from $\mathrm{G} 0$ mice. The affected transcripts were enriched in those involved in DNA damage responses, chromatin remodelling and histone modification: processes that are known to be altered in MDS. Therefore, DNA damage that activates the ATR pathway somehow alters the expression of splicing factors, and this suppresses erythroid differentiation of CMPs, leading to MDS.

Mutations in RNA splicing factors, such as SRSF2 and U2AF1, are associated with MDS, and two papers examined how these might influence MDS development. Kim, Ilagan,

Liang, Daubner et al. used

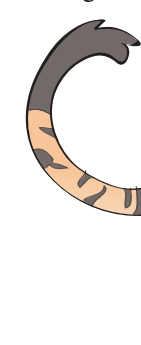

these papers demonstrate the importance of RNA splicing at different stages of MDS development

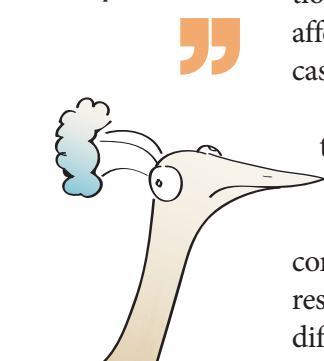

mice in which SRSF2-P95H (an MDSassociated mutation) was inducibly expressed in haematopoietic stem and progenitor cells. These mice had features of MDS (although none progressed to AML), including reduced levels of early erythroid progenitors. Furthermore, the $\mathrm{P} 95 \mathrm{H}$ mutation alters sequence specificity for the recognition motif to which SRSF2 binds in pre-mRNAs. Therefore, specific groups of transcripts are affected by the expression of SRSF2-P95H. For example, SRSF2-mutant cells harboured a specific mis-splicing event in $E Z H 2$ that resulted in nonsensemediated decay of the $E Z H 2$ transcript and consequent global downregulation of EZH2 expression.

Shirai et al. focused on U2AF1 mutations, which are thought to have a role in MDS initiation. Mice with bone marrow expressing the most common mutation, U2AF1-S34F, had leukopenia, as well as reduced frequency of bone marrow monocytes and B cells and an increased frequency of neutrophils and myeloid progenitors: CMPs and, variably, GMPs; however, these mice did not develop MDS or AML. Focusing on the increased frequency of CMPs, these authors used RNA sequencing to identify U2AF1-S34F-induced splicing alterations and found that changes mostly affected 3 ' splice acceptor sites and cassette exon events.

Together these papers demonstrate the importance of RNA splicing at different stages of MDS development and highlight an intriguing connection between DNA damage responses, RNA splicing and differentiation in the myeloid lineage.

Gemma K. Alderton

ORIGINAL RESEARCH PAPERS Colla, S. et al. Telomere dysfunction drives aberrant hematopoieti differentiation and myelodysplastic syndrome. Cancer Cell 27, 644-657 (2015) |Kim, E. et al. SRSF2 mutations contribute to myelodysplasia by mutantspecific effects on exon recognition. Cancer Cell 27, 617-630 (2015) |Shirai, C. et al. Mutant U2AF1 expression alters hematopoiesis and pre-mRNA splicing in vivo. Cancer Cell 27, 631-643 (2015) 